\title{
A Compact Diplexer with a Split-Ring Resonator Junction
}

\author{
Eugene A. Ogbodo, Yun Wu, Peter Callaghan, Yi Wang* \\ Department of Engineering Science, University of Greenwich (Medway Campus), Kent, ME4 4TB, U.K. \\ *Yi.Wang@gre.ac.uk
}

\begin{abstract}
Diplexers are three-port filtering devices with a junction connecting two channel filters; the common port connects to an antenna which serves the two channel filters bearing the second and the third port. In the design of diplexers, the technique used in connecting the two channel filters is of great importance to the designers. This is because it determines the size, the effectiveness of signal splitting and combining as well as the ease in implementation. In this paper, an asynchronously tuned double split-ring resonator (SRR) was used in joining the two channel filters. Without incurring any extra space for the junction, the SRR, being a dual-mode resonator, also functioned as a resonant pole for each channel filter. A diplexer operating at $2 \mathrm{GHz}$ and $3 \mathrm{GHz}$ with $4 \%$ fractional bandwidths and $20 \mathrm{~dB}$ return loss has been demonstrated. The interactions between the SRR and the channel filters were investigated. Good agreement between the simulated and the measured responses was achieved.
\end{abstract}

Keywords-Split Ring Resonator, Asynchronous coupling, Synchronous coupling

\section{INTRODUCTION}

Due to increasing demand in the communication system, there is need for robust and efficient devices to meet the traffic needs. A diplexer is one such device. It is conventionally designed by joining two channel filters using transmission line based junctions, hybrid couplers, manifolds or circulators [1-5]. Recently, resonant junction based techniques were introduced [6]. This resulted in new diplexer topologies [7-9] and reduction in circuit size [10-11]. A diplexer with a resonant junction tends to be more compact in size because the resonant junction stands as a resonating pole for each channel filer as well as a signal splitter and combiner. The transmission-line based signal-distribution network is no longer required. Such techniques have been reported in [11] where two novel multiplexer topologies based on all-resonator structures were implemented at X-band using waveguide technology. In [12], a diplexer with a resonant star-junction and a triplexer with a non-resonant node star-junction were presented using a synthesis approach. [13] presented a diplexer with a split-ring resonator (SRR) as a common resonator and signal splitter. In [14], a diplexer with a T-shaped junction resonator was presented. By extending the technique in [14] using open-loop resonators, a multiplexer and switchable diplexer were designed [15].

This work presents a diplexer that uses an asynchronously tuned SRR as a common resonator to connect two channel filters. The SRR comprises two nested open-loop resonators resonating at two different frequencies corresponding to the two passbands of the diplexer. This differs with [13] in that the nested resonators there are synchronously tuned, whereas these are asynchronously tuned in this work resulting in more widely separated channels. The two channels at $2 \mathrm{GHz}$ and $3 \mathrm{GHz}$ each with a $4 \%$ fractional bandwidth were separately designed and joined together using the SRR. The SRR contains the first resonator of each channel filter, resulting in reduced circuit size. Very importantly this also removes the need of any separate junction structures either based on transmission lines or resonators. It allowed the SRR to be effectively merged into the channel filters. Fig. 1 shows the difference between a conventional diplexer and the proposed diplexer topology.

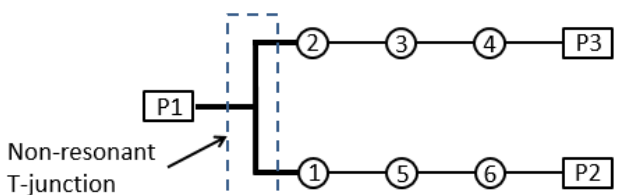

(a)

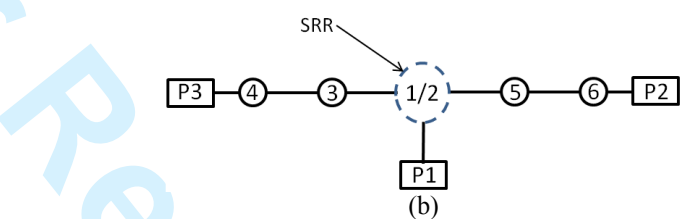

Fig. 1. A diplexer with six resonant poles: (a) Conventional topology; (b) Proposed topology

\section{DESIGN}

\section{A. Split Ring Resonator (SRR)}

Fig. 2 illustrates the proposed diplexer layout and its dimensions. The diplexer consists of one SRR and two sets of open-loop resonators. Each passband was designed to meet the specifications of a fractional bandwidth (FBW) of $4 \%$, and passband return loss of $20 \mathrm{~dB}$. Using the three-pole Chebyshev low-pass prototype derived from $[16,17]$ with $\mathrm{g}$ values of $\mathrm{g}_{0}=$ $\mathrm{g}_{4}=1.0, \mathrm{~g}_{1}=\mathrm{g}_{3}=0.8516$ and $\mathrm{g}_{2}=1.1032$, the coupling coefficient and Q-factors for the physical dimensioning of the resonators can be obtained using (1) and (2).

$$
\begin{aligned}
& M_{2,3}=M_{3,4}=M_{1,5}=M_{5,6}=\frac{F B W}{\sqrt{g_{1} g_{2}}}=0.041 \\
& Q_{e x 1}=Q_{e x 2}=Q_{e x 3}=\frac{g_{1} g_{0}}{F B W}=21.29
\end{aligned}
$$




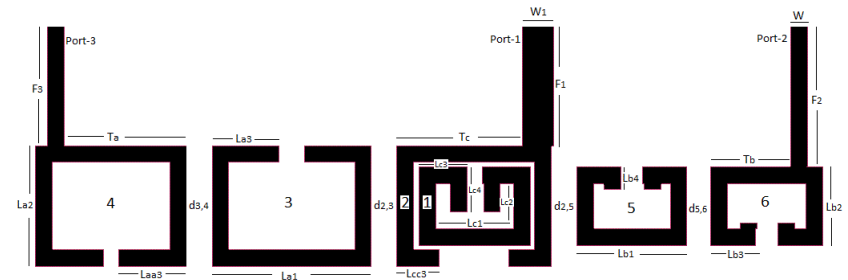

Fig. 2. Diplexer layout. F1 $=7.9 \mathrm{~mm}, \mathrm{~F} 2 \stackrel{\text { ㄴ }}{=} \overline{9.3 \mathrm{~mm}}, \mathrm{~F} 3=7.9 \mathrm{~mm}, \mathrm{Ta}=8.1$ $\mathrm{mm}, \mathrm{Tb}=5.3 \mathrm{~mm}, \mathrm{Tc}=8.3 \mathrm{~mm}, \mathrm{La} 1=10.2 \mathrm{~mm}, \mathrm{La} 2=8 \mathrm{~mm}, \mathrm{La} 3=4.5 \mathrm{~mm}$, Laa3 $=4.6 \mathrm{~mm}, \mathrm{Lb} 1=7.5 \mathrm{~mm}, \mathrm{Lb} 2=5.2 \mathrm{~mm}, \mathrm{Lb} 3=3.3 \mathrm{~mm}, \mathrm{Lcc} 3=2.9 \mathrm{~mm}$, $\mathrm{Lc} 1=7.6 \mathrm{~mm}, \mathrm{Lc} 2=5.2 \mathrm{~mm}, \mathrm{Lc} 3=3.3 \mathrm{~mm}, \mathrm{Lc} 4=3.1 \mathrm{~mm}, \mathrm{~d} 2,3=0.5 \mathrm{~mm}$, $\mathrm{d} 3,4=0.8 \mathrm{~mm}, \mathrm{~d} 2,5=0.7 \mathrm{~mm}, \mathrm{~d} 5,6=0.8 \mathrm{~mm}, \mathrm{~W}=1.2 \mathrm{~mm}, \mathrm{~W} 1=2.1 \mathrm{~mm}, \mathrm{~S}$ $=0.2 \mathrm{~mm}$

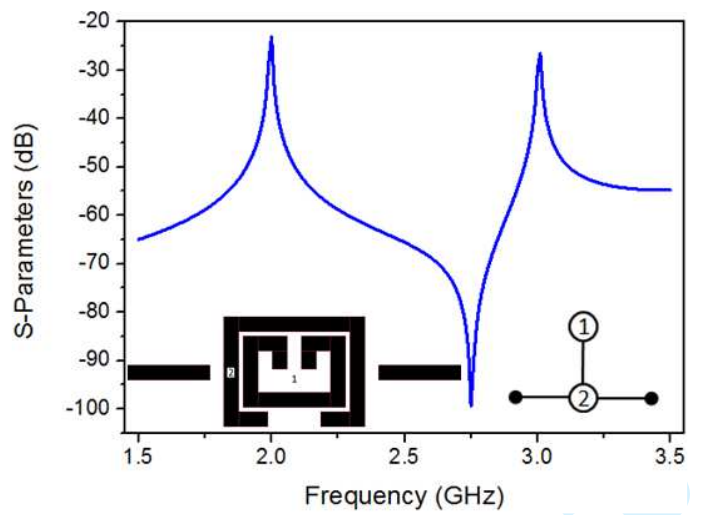

(a)

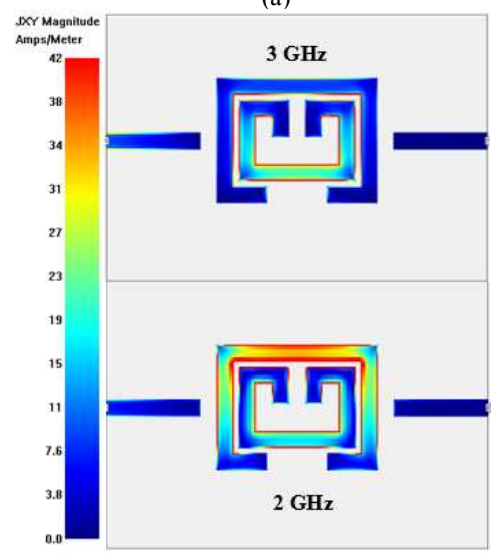

(b)

Fig. 3. SRR coupling topology: (a) response; (b) current distribution.

The two separately designed channel filters were then joined together using the SRR. In order to maintain the threepoles of the channel filters, the SRR acts as the first resonators of the two channel filters. This led to a reduction in the size of the diplexer. According to the diplexer topology of Fig. 1(b), the channel filters of the high passband is composed of resonators 1, 5 and 6 with resonator 1 being the inner ring of the SRR. Resonators 2, 3 and 4 represent the low passband channel filter with resonator 2 being the outer ring of the SRR. A cross-coupling is also established between resonator 1 of the SRR and resonator 3 and 5 of the two channel filters. This cross-coupling resulted in the introduction of additional transmission zeros in the diplexer $[13,18]$. The SRR is made up of two nested open-loop resonators of $2 \mathrm{GHz}$ and $3 \mathrm{GHz}$ which are coupled to each other. As shown in Fig. 3(a), the
SRR produced an inter-band transmission zero, which is a very useful feature in increasing the isolation between the two bands of the diplexer. The difficulty working with this resonator is that only the outer loop can be easily coupled with adjacent resonators or the feed lines [19]. Fig. 3(b) shows the current distributions at the two resonant frequencies.

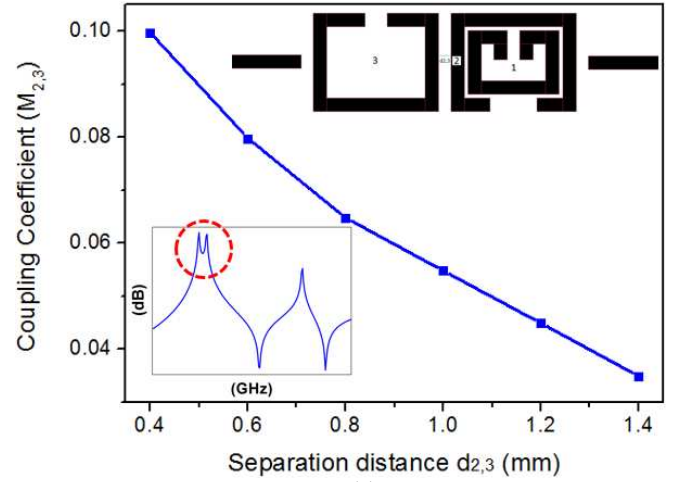

(a)

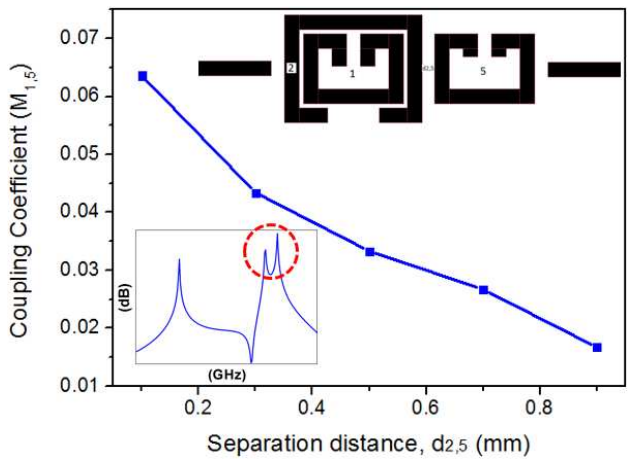

(b)

Fig. 4. (a). $M_{2,3}$ against $d_{2,3}$ with the inset of topology and typical response; (b) $M_{1,5}$ against $d_{2,5}$ with the inset of topology and typical response.

\section{B. Couplings}

The required coupling coefficient between the low passband channel filter and the SRR is achieved by adjusting the coupling distance $d_{2,3}$. Fig. 4 (a) illustrates the coupling coefficient $\mathrm{M}_{2,3}$ as a function of $\mathrm{d}_{2,3}$ with the inset showing a typical response. It should be noted that resonator 1 was present in the simulation to represent its potential loading effect. To couple the channel filter of high passband to the SRR, the change of coupling coefficient $\mathrm{M}_{1,5}$ as a function of $\mathrm{d}_{2,5}$ is investigated when resonator 2 is present, as shown in Fig. 4(b). It is worth noting that resonator 2 enhanced the coupling between resonator 1 and 5 . Without it, the coupling would be very weak.

Fig. 5 shows the resonant responses of the four resonators $1,2,3$ and 5 when coupled together. These achieved peaks of the eigen-modes can also be used in finding the coupling coefficients of $\mathrm{M}_{2,3}$ and $\mathrm{M}_{1,5}$. Again, it can be observed that the inter-band transmission zero as in Fig. 3 is still preserved but at a lower frequency due to the adjacent open loop resonators by the SRR. To determine the Q-factor for the common port, the coupling arrangement shown in Fig. 6 was used. The feed-line at port-1 was tapped to the SRR while the feed-lines at port-2 
and port- 3 were weakly coupled to the adjacent resonator. The common feed-line was adjusted both in position and width to achieve the required external couplings at both 2 and $3 \mathrm{GHz}$. (3) was used for the calculation of the Q-factor.

$$
Q_{e x}=\frac{f_{0}}{\Delta f}
$$

Where $\Delta f$ is the $3-\mathrm{dB}$ bandwidth from the peak of the resonance curves and $f_{0}$ represents the centre frequency of the resonance curves.

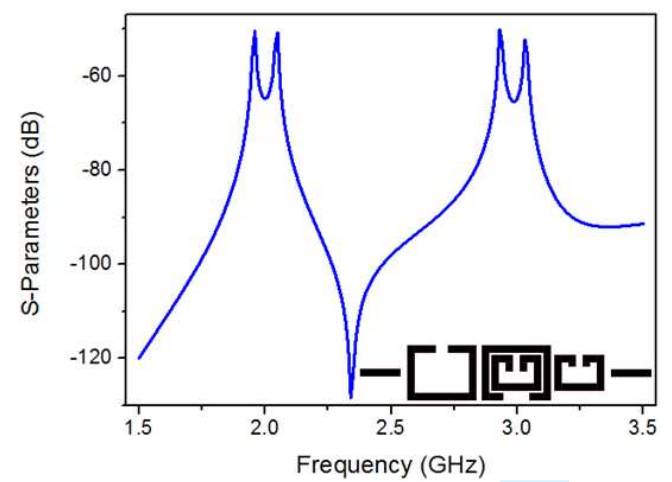

Fig. 5. Eigen-mode response and topology

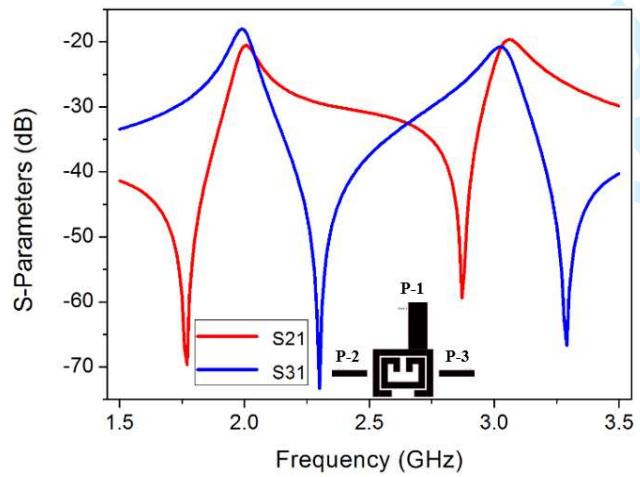

Fig. 6. Topology and typical response used to extract external Q-factors

\section{Diplexer Design}

The diplexer design started by firstly investigating the behaviours of each channel filter with the SRR. The first resonator of each channel filter was replaced with the SRR and the simulated response observed. Using dashed lines, Fig. 7 and 8 present the simulated responses achieved for the $2 \mathrm{GHz}$ and $3 \mathrm{GHz}$ band respectively. It can be observed that a transmission zero at $2.45 \mathrm{GHz}$ occurs above the lower passband of the filter in Fig. 7 whereas a transmission zero at $2.83 \mathrm{GHz}$ occurs below the upper band of the filter in Fig. 8 .

The two separately designed channel filters were then joined together using the SRR as a common resonator, followed by optimisation to meet the design specifications. Fig. 9 illustrates the simulated responses in dashed lines. The responses meet the design specifications of $20 \mathrm{~dB}$ return loss and FBW of 4\%, making the lower passband operate from 1.96 $\mathrm{GHz}$ to $2.04 \mathrm{GHz}$ and the higher passband operate from 2.94 $\mathrm{GHz}$ to $3.06 \mathrm{GHz}$. It is also noted that the two transmission zeros observed in the two separate channel filters are preserved within the guard band of the diplexer. Fig. 10 shows the simulated current distribution of the two channels during operation at $2 \mathrm{GHz}$ and $3 \mathrm{GHz}$. It is evident that the SRR operates as a signal splitter and combiner for the two channel filters.

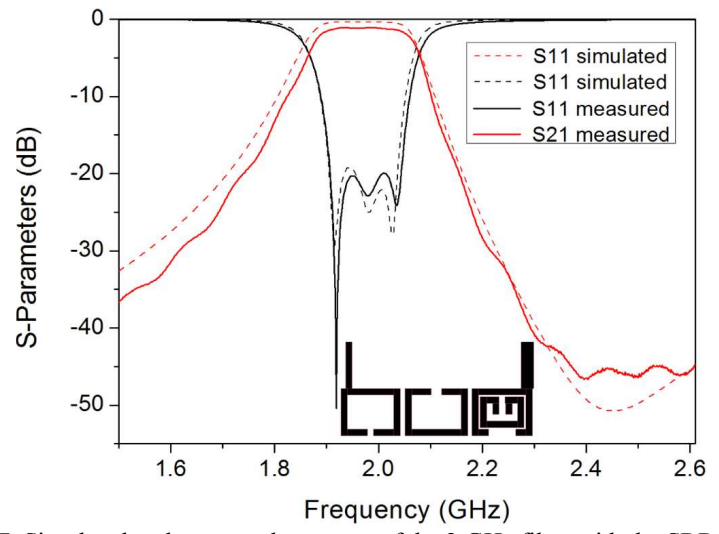

Fig. 7. Simulated and measured response of the $2 \mathrm{GHz}$ filter with the SRR.

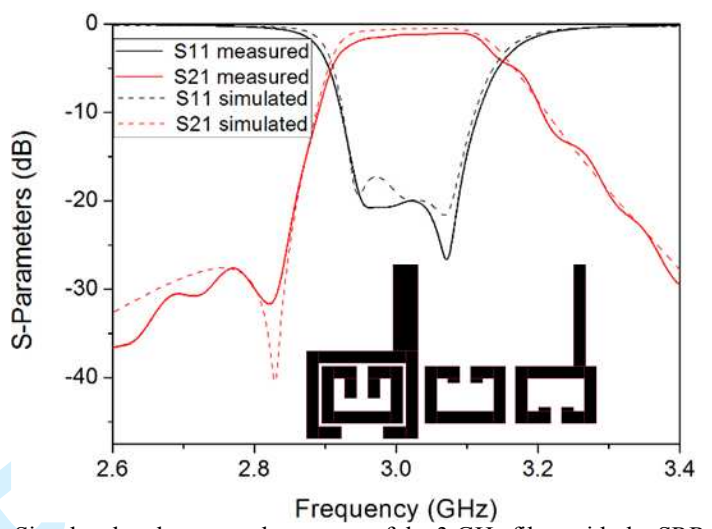

Fig. 8. Simulated and measured response of the $3 \mathrm{GHz}$ filter with the SRR

\section{FABRICATION AND MEASUREMENT}

The individual channel filters and the diplexer were all fabricated on Rogers 3010 substrate with a thickness of 1.27 $\mathrm{mm}$, a relative permittivity of 10.2 and loss tangent of 0.002 . Prototypes were fabricated using a LPKF ProtoMat S63 micro milling process and then measured using an Agilent Vector Network Analyser model N5230A. Fig. 11 shows the fabricated diplexer circuit. The measured responses are displayed in Fig. 7 to 9 (solid lines) for comparison against the simulated responses. It can be seen that measurements and simulations are in good agreement with each other. Fig. 7 showed that the measured response slightly shifted up by about $5 \mathrm{MHz}$, the insertion loss was about $2 \mathrm{~dB}$ and the return loss was at $20 \mathrm{~dB}$. Fig. 8 showed that the measured response maintained the same bandwidth with the simulated response. The insertion loss is about $2 \mathrm{~dB}$ while the return loss is about $20 \mathrm{~dB}$. For the diplexer response shown in Fig. 9, the measured response has its low passband frequency shifted to the higher by about $5 \mathrm{MHz}$, the high passband frequency shifted by about $2 \mathrm{MHz}$. The return loss is $22 \mathrm{~dB}$ and $25 \mathrm{~dB}$ for the low and high passband respectively. The insertion loss is about $2 \mathrm{~dB}$ for both passbands. The small discrepancies were mainly due to 
fabrication tolerance and the variation of the dielectric constant for the high permittivity dielectric material.

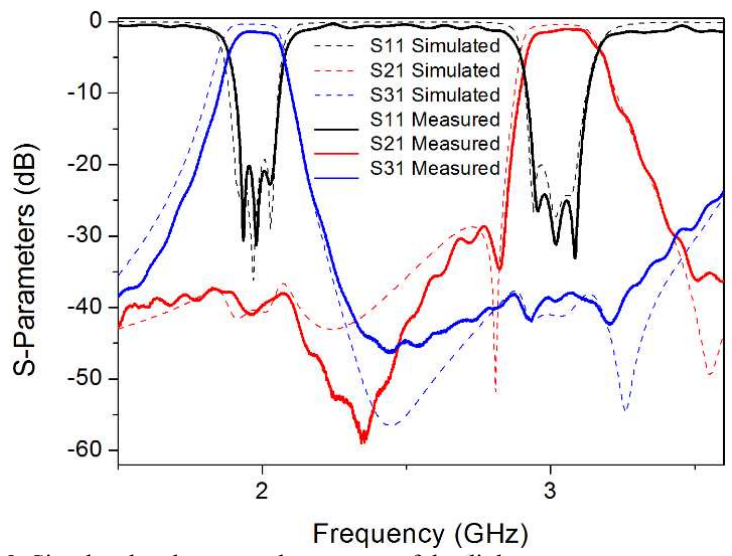

Fig. 9. Simulated and measured responses of the diplexer.

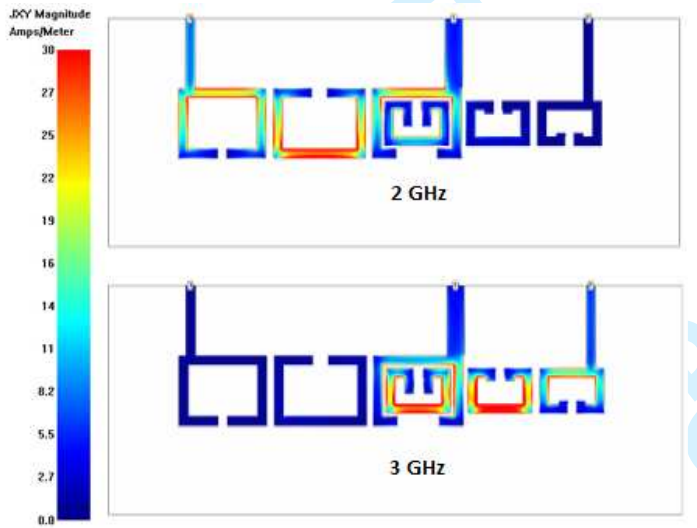

Fig. 10. Simulated current distribution of the diplexer at 2 and $3 \mathrm{GHz}$

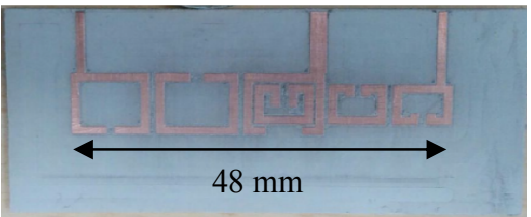

Fig. 11. Photo of the fabricated diplexer.

\section{CONCLUSION}

In this paper, a diplexer with a compact junction structure using a SRR was designed to operate at $2 \mathrm{GHz}$ and $3 \mathrm{GHz}$ passbands. It made use of an asynchronously tuned SRR as its common resonator joining two separately designed channel filters. By using the asynchronously tuned SRR, a diplexer having widely-separated channels was realised. The SRR, being a dual-mode resonator, acted as a signal splitter and combiner for the two channels and at the same time as a resonant pole for each channel. The proposed all-resonator based diplexer achieved three poles for each channel using four single-mode resonators and one dual mode SRR. This resulted in a reduced circuit size as the need for the conventional transmission-line based junction is eliminated.

\section{REFERENCES}

[1] R. Cameron, M. Yu, "Design of manifold-coupled multiplexers," IEEE Microwave Magazine, vol.8, no.5, pp.46-59, 2007.

[2] T. Yang, P-L. Chi and T. Itoh, "High Isolation and Compact Diplexer Using the Hybrid Resonators," IEEE Microwave and Wireless Components. Letters, Vol. 20, No. 10, Oct. 2010.

[3] C. Chen, H. Wu and W. Wu, "High Isolation DBR Diplexer Using InLine SCMRC", Progress In Electromagnetics Research C, Vol. 22, 97108, 2011.

[4] T.F. Skaik, M.J. Lancaster, F. Huang, "Synthesis of multiple output coupled resonator circuits using coupling matrix optimisation," Microwaves, Antennas \& Propagation, IET, vol.5, no.9, pp.1081-1088, June 272011.

[5] S. Bastioli, L. Marcaccioli and R. Sorrentino, "An Original Resonant Yjunction for compact Waveguide Diplexers," Proceeding of IEEE MTTS International Microwave Symposium Digest, Boston, 7-12 June 2009, pp. 1233-1236.

[6] W. Xia, X. Shang and M. J. Lancaster, "All-resonator-based waveguide diplexer with cross-couplings," in Electronics Letters, vol. 50, no. 25, pp. 1948-1950, 1242014.

[7] R. Wang and J. Xu, "Synthesis and design of microwave diplexers with a common resonator junction," Microwave and Millimeter Wave Technology (ICMMT), 2012 International Conference on, Shenzhen, 2012, pp. 1-4.

[8] D. A. Tubail and T. F. Skaik, "Synthesis of coupled resonator-based multiplexers with generalised structures using coupling matrix optimisation," in Electronics Letters, vol. 51, no. 23, pp. 1891-1893, 11 52015.

[9] Y. Wang and M. J. Lancaster, "An Investigation on the coupling characteristics of a novel multiplexer configuration," Microwave Conference (EuMC), 2013 European, Nuremberg, 2013, pp. 900-903.

[10] H. Ezzeddine, S. Bila, S. Verdeyme, F. Seyfert and D. Pacaud, "Coupling topologies for realizing compact microwave diplexers with dual-mode cavities," Microwave Symposium Digest (MTT), 2010 IEEE MTT-S International, Anaheim, CA, 2010, pp. 880-883.

[11] X. Shang, Y. Wang, X. Wenlin, M.J. Lancaster, "Novel Multiplexer Topologies Based on All-Resonator Structures," IEEE Trans. Microwave Theory and Techniques, vol 61, no. 11, 2013, pp. $3838-$ 3845 .

[12] P. Zhao and K. L. Wu, "An Iterative and Analytical Approach to Optimal Synthesis of a Multiplexer With a Star-Junction," IEEE Transactions on Microwave Theory and Techniques, vol. 62, no. 12, pp. 3362-3369, Dec. 2014

[13] G.-L. Alejandro, G.-G. Roberto, S.-P. Magdalena, "Compact diplexer with edge-coupled and nonbianisotropic split-ring resonators," Microwave Symposium Digest (MTT), 2012 IEEE MTT-S international, vol., no., pp.1-3, 17-22 June 2012.

[14] M. L. Chuang and M. T. Wu, "Microstrip diplexer design ussing common T-shaped resonator," IEEE Microwave. Wireless Components. Letter., vol. 21, no. 11, pp. 583-585, Nov. 2011.

[15] M. L. Chuang and M. T. Wu, "Microstrip Multiplexer and Switchable Diplexer with Joint T-Shaped Resonator," IEEE Microwave and Wireless Components. Letters, Vol. 24, No. 5, May 2014.

[16] J.-S. Hong "Microstrip Filters for RF/Microwave Applications," John Wiley \& Sons, Inc. 2011.

[17] D. M. Pozar, Microwave Engineering. 3rd ed. New York: Wiley, 2005.

[18] S. Amari, M. Bekheit and F. Seyfert, "Notes on bandpass filters whose inter-resonator coupling coefficients are linear functions of frequency," Microwave Symposium Digest, 2008 IEEE MTT-S International, Atlanta, GA, USA, 2008, pp. 1207-1210.

[19] A. G. Lamperez and M. S. Palma, "Dual band filter with split-ring resonators," Microwave Symposium Digest, 2006. IEEE MTT-S International, San Francisco, CA, 2006, pp. 519-522. 